\title{
Spin Transfer due to Quantum Magnetization Fluctuations
}

\author{
Andrei Zholud, Ryan Freeman, Rongxing Cao, Ajit Srivastava, and Sergei Urazhdin \\ Department of Physics, Emory University, Atlanta 30322, Georgia, USA
}

(Received 15 May 2017; revised manuscript received 25 August 2017; published 18 December 2017)

\begin{abstract}
We utilize a nanoscale magnetic spin-valve structure to demonstrate that current-induced magnetization fluctuations at cryogenic temperatures result predominantly from the quantum fluctuations enhanced by the spin transfer effect. The demonstrated spin transfer due to quantum magnetization fluctuations is distinguished from the previously established current-induced effects by a nonsmooth piecewise-linear dependence of the fluctuation intensity on current. It can be driven not only by the directional flows of spinpolarized electrons, but also by their thermal motion and by scattering of unpolarized electrons. This effect is expected to remain non-negligible even at room temperature, and entails a ubiquitous inelastic contribution to spin-polarizing properties of magnetic interfaces.
\end{abstract}

DOI: 10.1103/PhysRevLett.119.257201

Spin transfer [1-3] - the transfer of angular momentum from spin-polarized electrical current to magnetic materials - has been extensively researched as an efficient mechanism for the electronic manipulation of nanomagnetic systems, advancing our understanding of nanomagnetism and electronic transport, and enabling the development of magnetoelectronic nanodevices [3-15]. This effect can be understood based on the argument of angular momentum conservation for spin-polarized electrons, scattered by a ferromagnet whose magnetization $\vec{M}$ is not aligned with their polarization. The component of the electron spin transverse to $\vec{M}$ becomes absorbed, exerting a spin transfer torque (STT) on the magnetization. In nanomagnetic devices such as spin valve nanopillars [Fig. 1(a)], STT can enhance thermal fluctuations of magnetization [Fig. 1(b)], resulting in its reversal $[5,16]$ or auto-oscillation [6], which can be utilized in memory, microwave generation, and spin-wave logic $[17,18]$.

The approximation for the magnetization as a thermally fluctuating classical vector $\vec{M}$ provides an excellent description for the quasiuniform magnetization dynamics [19]. However, for typical transition-metal ferromagnets, the frequencies $f$ of the dynamical magnetization modes extend to $\sim 100 \mathrm{THz}[19,20]$. The modes with $f>6 \mathrm{THz}$ are frozen out at room temperature $(f>70 \mathrm{GHz}$ at $T=3.4 \mathrm{~K}$ ), and the effect of STT on them cannot be described in terms of the enhancement or suppression of thermal fluctuations. Such high-frequency modes are only now becoming experimentally accessible [21-24], and their role in spin transfer remains unexplored.

Here, we introduce a frequency nonselective, magnetoelectronic approach allowing measurements of the effects of spin transfer on the magnetization fluctuations, not limited to quasiuniform modes. Our central result is that at low temperatures the current-dependent fluctuations arise predominantly from the quantum fluctuations enhanced by spin transfer; the quantum contribution remains non-negligible even at room temperature. The observed effect is analogous to the well-studied spontaneous emission of a photon by a two-level system, also caused by quantum fluctuations, which occurs even when there are no photons to stimulate the emission. In the (a)

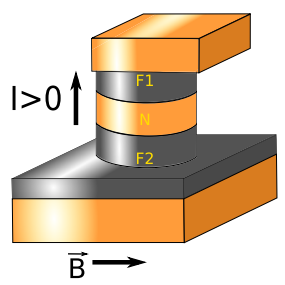

(c)

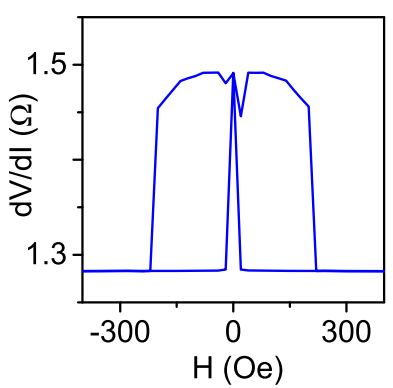

(b)
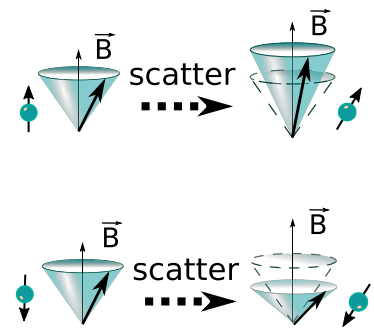

(d)

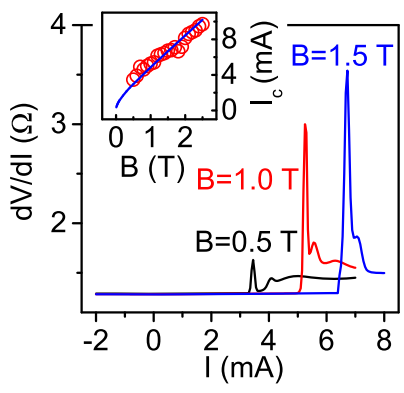

FIG. 1. (a) Schematic of the tested spin-valve nanopillar. Gray: Permalloy (Py) free layer $F 1$ and polarizer $F 2$. Orange: $\mathrm{Cu}$ electrodes and spacer $N$. (b) Effect of STT on thermal magnetization fluctuations. Top (bottom): Spin transfer due to scattering of the majority (minority) electron by the magnetization results in a decrease (increase) of thermal fluctuations. Vectors show the direction of the fluctuating magnetization and the magnetic moment of the scattered electron. (c) Magnetoelectronic hysteresis loop. (d) Differential resistance vs current, at the labeled fields. Inset: Critical current $I_{c}$ for the onset of auto-oscillation vs field determined from the experimental data (symbols), and the calculation (curve). All measurements were performed at 3.4 K. 
studied magnetic system, the role of photons is played by magnons - the quanta of the dynamical magnetization modes.

We can describe the effects of STT on thermal magnetization fluctuations [Fig. 1(b)] in terms of the currentdependent fluctuation energy, or, equivalently, the population of magnons [2,25],

$$
\left\langle N^{(\mathbf{i})}\right\rangle=\frac{N_{0}^{(\mathbf{i})}}{1-I / I_{c}^{(\mathbf{i})}},
$$

where $\mathbf{i}$ enumerates the magnon mode, $N_{0}^{(\mathbf{i})}$ is its equilibrium population, and $I_{c}^{(\mathbf{i})}$ is the critical current for the onset of the dynamical instability [1-3]. We note that Eq. (1) is valid only for $I<I_{c}$; for $I>I_{c}$ the magnon population is determined by the nonlinear dynamical mechanisms not captured by this equation. The dependence Eq. (1) has been verified by magneto-optical [25] and magnetoelectronic techniques [26]. We utilized the latter to verify the established effects of STT in the spin-valve nanopillars used in our study. The dependence of resistance on the magnetic field $B$ for our test structure is typical for the giant magnetoresistance (GMR) [27] in magnetic nanopillars, Fig. 1(c). The current-dependent differential resistance exhibits a peak due to the onset of the dynamical instability at $I_{c}$ [5-7], Fig. 1(d). The dependence $I_{c}(B)$ agrees with the calculation based on the spin torque theory [1] using the Kittel formula for the frequency of auto-oscillation [28], inset in Fig. 1(d).

To introduce our approach to magnetoelectronic measurements of current-dependent magnetization fluctuations, we analyze the relationship between GMR and the magnon population. The dependence of resistance on the angle $\theta$ between the magnetizations of the "free" layer $F 1$ and the "polarizer" $F 2$ [Fig. 1(a)] is $R(\theta)=R(0)+\Delta R \sin ^{2}(\theta / 2) \approx$ $R(0)+\Delta R \theta^{2} / 4$, with magnetoresistance $\Delta R$ [29]. The current-induced fluctuations discussed below are dominated by the nonuniform dynamical states. A quadratic relationship $R(\theta) \approx R(0)+C \Delta R \theta^{2} / 4$ is also expected for such states, with the coefficient $C$ of order 1 reflecting the contribution from electron diffusion across magnetically inhomogeneous regions.

To analyze the relation between $\theta$ and the magnon population in $F 1$, we note that a magnon in any mode has spin 1 [20]. Therefore, for a ferromagnet with the total spin $S=M V / 2 \mu_{B}$, the total magnon population is $N=$ $M V \sin ^{2}(\theta / 2) / \mu_{B}[30]$. Here, $M$ is magnetization, and $V$ is volume. Resistance is therefore proportional to the total magnon population, $R(\theta)=R(0)+C N \mu_{B} \Delta R / M V$. Thus, the current dependence of resistance reflects the variations of the total magnon population in the free layer $F 1$, not limited to quasiuniform dynamical modes. Here, we neglect the effects of current on the polarizer F2. It was thicker and only partially patterned to allow the magnons generated due to spin transfer to escape from the active area.

To determine the effect of spin transfer on the fluctuation intensity in the studied spin-valve nanopillars, we analyze the differential resistance at subcritical currents, Fig. 2(a). The current dependence exhibits an unusual piecewiselinear shape, with the slope larger for $I>0$ than for $I<0$. The curves are shifted by the field, which can be explained by the magnon freeze-out, as illustrated in Fig. 2(b), which shows the field dependence of resistance at $I=0$ together with the calculated field-dependent thermal magnon population [30]. Since field variation does not noticeably affect the slopes of the curves in Fig. 2(a), the observed piecewise-linear dependence cannot be associated with thermal fluctuations whose intensity is controlled by the field. It cannot be explained by Joule heating, because the (a)

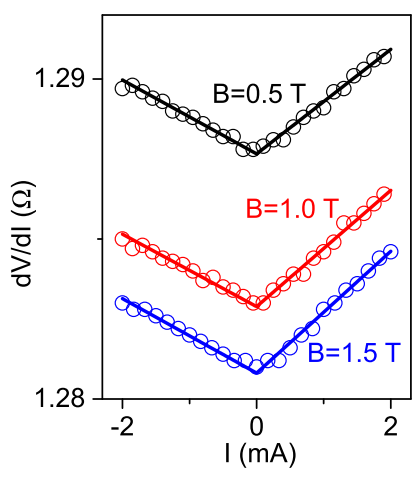

(b)

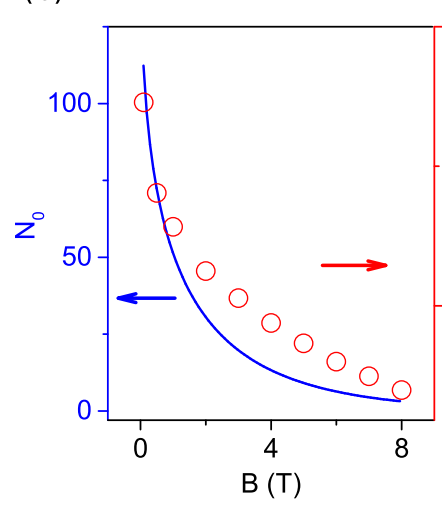

(c)

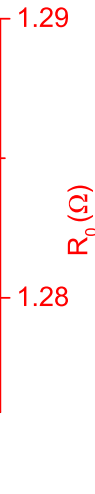

(d)

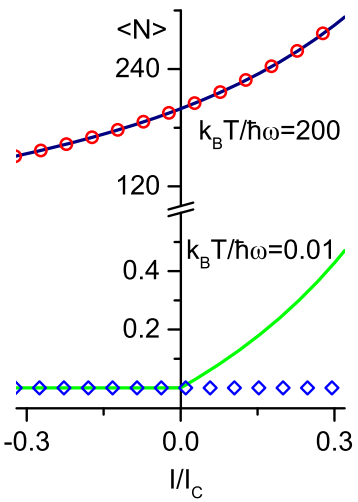

FIG. 2. (a) Differential resistance vs current, at the labeled values of field and $T=3.4 \mathrm{~K}$. Symbols, data; lines, best linear fits performed separately for $I<0$ and $I>0$. (b) Differential resistance (symbols, right scale) and the calculated total magnon population (curve, left scale) vs $B$ at $I=0$. (c) Scattering of the majority (top) and the minority (bottom) electron by magnetization experiencing only quantum fluctuations. Vectors show the direction of the fluctuating magnetization and the magnetic moment of the scattered electron. (d) Population of the magnon mode with frequency $\omega$ vs current, in the classical limit $k_{B} T / \hbar \omega=200$ and in the quantum limit $k_{B} T / \hbar \omega=0.01$, for $p=1$. Symbols, classical result based on Eq. (1); curves, quantum result based on Eq. (3). 
dissipated power, and thus the resulting resistance increase, is quadratic in current. It is also inconsistent with the analytical expression Eq. (1) of spin torque theory. Electronic shot noise exhibits a similar linear increase of power with bias [37]. However, shot noise (fluctuating current) can contribute to the differential resistance only by inducing magnetization fluctuations, which in the absence of thermal fluctuations is forbidden by the angular momentum conservation argument of spin torque theory.

We conclude that a previously unrecognized contribution to spin transfer, not described as enhancement of thermal magnetization fluctuations, results in a linear increase of magnon population with current. To interpret our observations, we note that even if thermal fluctuations are negligible at low temperature, the polarization of the scattered electrons cannot be perfectly aligned with the magnetization because of the quantum fluctuations of the latter, leading to electron spin dynamics driving spin transfer. The proposed quantum effect must be distinct from STT described by Eq. (1). In particular, in contrast to thermal fluctuations, quantum fluctuations cannot be suppressed by scattering of the majority electrons, due to the uncertainty principle [Fig. 2(c), top]. However, they can be enhanced by scattering of the minority electrons [Fig. 2(c), bottom]. To satisfy angular momentum conservation, the transverse to the field component of the magnetization must remain zero. The resulting substantially nonclassical magnetization state cannot be described by rotation from the initial state, and therefore the proposed effect is not a torque.

Despite the ongoing work [38-40], there is no established quantum theoretical framework for spin transfer. We can describe the uniform (FMR) mode by the dynamical states of a quantum macrospin $\vec{S}$ representing the magnetization [41]. Its projection $S_{z}$ on the equilibrium direction (opposite to $\vec{B}$ ) characterizes the magnon population $N=S-S_{z}$. An electron with spin $\vec{s}=(a, b)$, where $a$ and $b$ are complex and $|a|^{2}+|b|^{2}=1$, interacts with the magnetic layer via the exchange interaction $H_{\mathrm{ex}}=J_{\mathrm{ex}} \vec{s} \cdot \vec{S} / S$, where $J_{\mathrm{ex}}$ is exchange energy. This results in the precession of both $\vec{S}$ and $\vec{s}$ around the total angular momentum $\vec{J}=\vec{S}+\vec{s}$ conserved by the exchange Hamiltonian. Following Slonczewski's argument [1], we assume that the precession phases are randomized due to variations among electron trajectories. At $N \ll S$, the change of $S_{z}$, and thus the average number $\langle\Delta N\rangle$ of magnons generated by the scattered electron is $[30,41]$,

$$
\langle\Delta N\rangle=-\left\langle\Delta S_{z}\right\rangle \approx \frac{|b|^{2}}{S}+\frac{|b|^{2}}{S} N-\frac{|a|^{2}}{S} N .
$$

This equation can be interpreted by analogy to the interaction between a two-level system and the electromagnetic field. The two-level system is the spin of the scattered electron, and the role of photons is played by magnons. The first term describes spontaneous emission of magnons, which occurs even in the absence of magnons at $N=0$. The second and third terms describe stimulated emission and absorption, respectively, with the probability proportional to the number of magnons [2].

In the steady state, the magnon population is determined by the balance between spin transfer driven by current $I$, and the dynamical relaxation. Describing the latter by Landau damping, or equivalently for small $N$ by the relaxation time approximation $\left.(\partial N / \partial t)\right|_{\text {rel }}=-\left(N-N_{0} / \tau\right)$ $[2,41]$ with $\tau=1 /(2 \alpha \omega)$, where $\omega$ is the FMR mode frequency, we obtain [30]

$$
\langle N(I)\rangle=\frac{N_{0}+(|I| / p+I) / 2 I_{c}}{1-I / I_{c}},
$$

where $p=|a|^{2}-|b|^{2}$ describes the current polarization. The unusual nonanalytical form of Eq. (3) originates from the asymmetry of Eq. (2) with respect to exchange of $a$ and $b$ describing current reversal. Note that Eq. (3) does not diverge at $p=0$, since $I_{c}=e S /(\tau \xi p) \propto 1 / p$ [30]. Here, $\xi$ is a coefficient of order 1 determined by the transport properties of the free layer.

In the classical limit $N_{0} \gg 1$, the spontaneous contribution in Eq. (2) is negligible, and Eq. (3) reduces to the STT result Eq. (1) [Fig. 2(d), top]. In the quantum limit $N_{0} \ll 1$ at $T \ll \hbar \omega^{(\mathbf{i})} / k_{B}$, we obtain a piecewise-linear dependence [bottom curve in Fig. 2(d)]. The data in Fig. 2(a) are consistent with the dominant quantum contribution once we account for the imperfect electron spin polarization, $p<1$ in Eq. (3), resulting in spontaneous magnon generation at both positive and negative currents. Based on Eq. (3), the ratio between the positive- and the negative-current slopes in the quantum limit is $(1+p) /(1-p)$, providing a new method for analyzing spin-polarizing properties of ferromagnets.

We now discuss the significance of the proposed quantum contribution to spin transfer. Since exchange interaction between the electron spin and the magnetization underlying spin transfer is local, Eq. (3) with modedependent values $N_{0}^{(\mathbf{i})}, I_{c}^{(\mathbf{i})}$, must be applicable not only to FMR, but also to the nonuniform modes [42]. The quasiuniform modes with frequency $f$ of a few $\mathrm{GHz}$ are degenerately populated at $3.4 \mathrm{~K}$; the contribution of their quantum fluctuations to spin transfer is negligible. However, the modes with $f>70 \mathrm{GHz}$ are frozen out. Summation of Eq. (3) over the magnon spectrum confirmed that the quantum contribution to the total current-dependent magnon population is dominant at $3.4 \mathrm{~K}$, consistent with our interpretation of Fig. 2(a) [30]. We note that for shortwavelength modes, additional resonant contributions may arise due to the interplay between magnetization dynamics and orbital electron motion; we leave their analysis to future studies. 
(a)

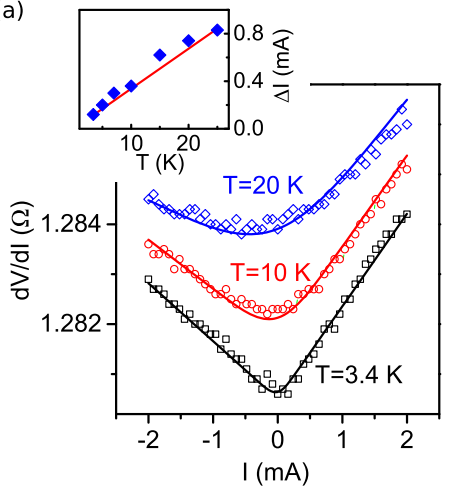

(b)
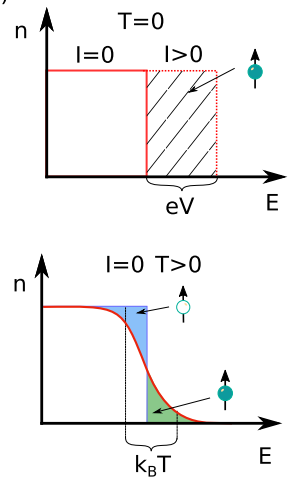

FIG. 3. (a) Symbols: differential resistance vs current at the labeled values of temperature and $B=1 \mathrm{~T}$. Curves: results of fitting with a piecewise-linear dependence convolved with a Gaussian whose width is used as an adjustable parameter. The data are offset for clarity. Inset: temperature dependence of the Gaussian width extracted from the fitting (symbols), and $\Delta I=$ $1.9 k_{B} T / e R_{0}$ (line). (b) Top: at $T=0$, the Fermi distribution of scattered electrons is steplike. Bias current shifts the distribution, driving the spin transfer. Bottom: at finite $T$, scattering of thermal electrons and holes occurs even at zero bias, equivalent to bias distribution of width $k_{B} T / e$.

At higher temperatures, the zero-current singularity becomes rapidly broadened [Fig. 3(a)]. This cannot be attributed to the increasing role of thermal magnetization fluctuations, since the piecewise-linear dependence is still apparent at larger currents even at $20 \mathrm{~K}$. To analyze this effect, we fit the data with a piecewise-linear dependence convolved with a Gaussian. The extracted broadening closely follows a linear dependence $\Delta I=(1.9 \pm 0.1) k_{B} T /$ $e R_{0}$, where $R_{0}$ is the resistance at $I=0$ [inset in Fig. 3(a)]. This result is consistent with the proposed quantum mechanism. Bias current shifts the electron distribution in the magnetic nanopillar, driving spin transfer [Fig. 3(b), top]. At finite temperature, the electron distribution becomes thermally broadened, resulting in scattering of thermally excited electrons and holes [Fig. 3(b), bottom] equivalent to a distribution of width $\Delta V=k_{B} T / e$ of the bias voltage applied to $F 1$, facilitating spin transfer even in the absence of directional current flow. The observed broadening is consistent with similar contributions of layers $F 1$ and $F 2$ to $R_{0}$, such that $\Delta V \approx I R_{0} / 2$. Thermal broadening is absent for STT, because the effects of electron and hole scattering cancel each other.

We now analyze the temperature dependence of spin transfer. We convolve Eq. (3) with a Gaussian, to account for thermal effects discussed above, and take a derivative with respect to current. At $I=0$, we obtain

$$
\left.\frac{d N^{(\mathbf{i})}}{d I}\right|_{I=0}=\frac{1}{2 I_{c}^{(\mathbf{i})}}+\frac{N_{0}^{(\mathbf{i})}}{I_{c}^{(\mathbf{i})}} .
$$

The first term on the right, describing the quantum contribution to the current-dependent magnon population,

is independent of temperature. The last term describing STT decreases with decreasing $T$, and vanishes at $T=0$. We define $T_{x}$ as the crossover temperature at which the two contributions become equal. Figure 4(a) shows the calculated temperature dependence of $d N / d I_{I=0}$ for the total magnon population at $B=1 \mathrm{~T}$, obtained by summing Eq. (4) over all the magnon modes. The increase with temperature associated with STT is approximately linear, indicating that the classical contribution is dominated by the degenerately populated modes described by the Rayleigh-Jeans law. Based on these calculations, $T_{x}=$ $126 \mathrm{~K}$ for the total magnon population. The measured $d R / d I$, which is proportional to $d N / d I$, increases linearly with temperature, with a nonzero $T=0$ intercept [Fig. 4(b)]. We use linear extrapolation to estimate $T_{x}=160 \mathrm{~K}$, in a good agreement with calculations. If a single mode were involved in STT, the value of $T_{x}$ would characterize its frequency $f_{0}=\ln (3) k_{B} T_{x} / h \approx 4 \mathrm{THz}$ [see Eq. (4)]. This result confirms that spin transfer involves high-frequency dynamical modes extending into the $\mathrm{THz}$ frequency range.

The presented results have significant implications for magnetoelectronic effects in a variety of magnetic systems. Quantum fluctuations can contribute to current-induced phenomena whenever highly nonuniform dynamical states are involved, for example, in reversal via domain wall motion [11]. Generally, the quantum magnon generation decreases the effective magnetization, lowering the reversal barriers. The contribution of quantum fluctuations to spin transfer in antiferromagnets [43] must be larger than in ferromagnets, due to higher magnon frequencies. Quantum fluctuations may contribute to other phenomena involving interaction between magnetization and conduction electrons, including spin pumping [44], spin-orbit [45-47], optically driven [48-50], and spin-caloritronic effects [51-55].

We also infer a significant inelastic contribution to spindependent electron transport in ferromagnets due to quantum electron-magnon scattering. It is presently believed that currents flowing through ferromagnets become spin
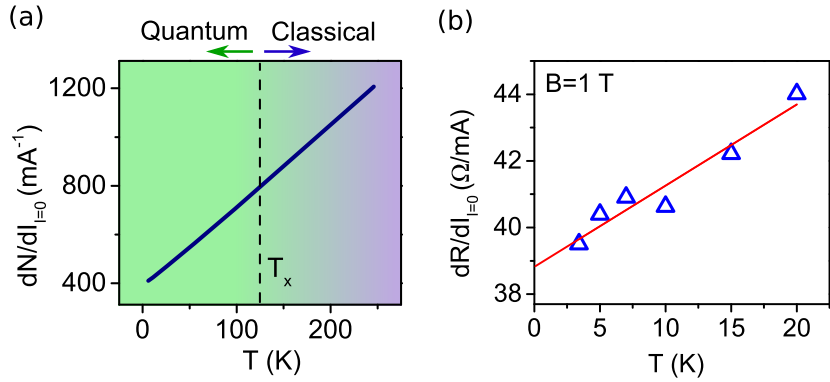

FIG. 4. (a) The calculated slope $d N / d I$ vs $T$, at $B=1 \mathrm{~T}$. Vertical dashed line marks the crossover temperature $T_{x}$ between the quantum and the classical spin transfer regimes. (b) Symbols: The slope $d R / d I$ of resistance vs current at $I=0$ and $B=1 \mathrm{~T}$. Line: best linear fit of the data. 
polarized mainly due to spin anisotropy of electron scattering. Meanwhile, according to Eq. (2), an unpolarized conduction electron scattered by the ferromagnet generates a magnon in a given dynamical mode with probability $1 / 2 S$, where $S$ is the total spin of the ferromagnet; it becomes majority spin polarized in this process. The number of modes is of order $S$ [19], and therefore the total probability for an initially unpolarized electron to become majority polarized due to quantum magnon generation is of order 1 . This result shows that inelastic scattering of electrons by quantum magnetization fluctuations provides a non-negligible contribution to spin-polarizing properties of ferromagnets.

We acknowledge support from the NSF Grants No. ECCS-1503878 and No. DMR-1504449.

[1] J. Slonczewski, J. Magn. Magn. Mater. 159, L1 (1996).

[2] L. Berger, Phys. Rev. B 54, 9353 (1996).

[3] D. Ralph and M. Stiles, J. Magn. Magn. Mater. 320, 1190 (2008).

[4] M. Tsoi, A. G. M. Jansen, J. Bass, W.-C. Chiang, V. Tsoi, and P. Wyder, Nature (London) 406, 46 (2000).

[5] J. A. Katine, F. J. Albert, R. A. Buhrman, E. B. Myers, and D. C. Ralph, Phys. Rev. Lett. 84, 3149 (2000).

[6] S. I. Kiselev, J. C. Sankey, I. N. Krivorotov, N. C. Emley, R. J. Schoelkopf, R. A. Buhrman, and D. C. Ralph, Nature (London) 425, 380 (2003).

[7] W. H. Rippard, M. R. Pufall, S. Kaka, S. E. Russek, and T. J. Silva, Phys. Rev. Lett. 92, 027201 (2004).

[8] Y. B. Bazaliy, B. A. Jones, and S.-C. Zhang, Phys. Rev. B 57, R3213 (1998).

[9] K.-J. Lee, A. Deac, O. Redon, J.-P. Nozières, and B. Dieny, Nat. Mater. 3, 877 (2004).

[10] A. A. Tulapurkar, Y. Suzuki, A. Fukushima, H. Kubota, H. Maehara, K. Tsunekawa, D. D. Djayaprawira, N. Watanabe, and S. Yuasa, Nature (London) 438, 339 (2005).

[11] S. Mangin, D. Ravelosona, J. A. Katine, M. J. Carey, B. D. Terris, and E. E. Fullerton, Nat. Mater. 5, 210 (2006).

[12] M. Madami, S. Bonetti, G. Consolo, S. Tacchi, G. Carlotti, G. Gubbiotti, F. B. Mancoff, M. A. Yar, and J. Åkerman, Nat. Nanotechnol. 6, 635 (2011).

[13] N. Locatelli, V. Cros, and J. Grollier, Nat. Mater. 13, 11 (2013).

[14] K.-S. Ryu, L. Thomas, S.-H. Yang, and S. Parkin, Nat. Nanotechnol. 8, 527 (2013).

[15] A. D. Kent and D. C. Worledge, Nat. Nanotechnol. 10, 187 (2015).

[16] S. Zhang, P. M. Levy, and A. Fert, Phys. Rev. Lett. 88, 236601 (2002).

[17] B. Behin-Aein, D. Datta, S. Salahuddin, and S. Datta, Nat. Nanotechnol. 5, 266 (2010).

[18] S. S. P. Parkin, M. Hayashi, and L. Thomas, Science 320, 190 (2008).

[19] A. G. Gurevich and G. A. Melkov, Magnetization Oscillations and Waves (CRC Press, Boca Raton, 1996).

[20] R. M. White, Quantum Theory of Magnetism (Springer Berlin Heidelberg, 2007).
[21] S. Bonetti, R. Kukreja, Z. Chen, F. Macià, J. M. Hernàndez, A. Eklund, D. Backes, J. Frisch, J. Katine, G. Malm, S. Urazhdin, A. D. Kent, J. Stöhr, H. Ohldag, and H. A. Dürr, Nat. Commun. 6, 8889 (2015).

[22] S. Bonetti, M. Hoffmann, M.-J. Sher, Z. Chen, S.-H. Yang, M. Samant, S. Parkin, and H. Dürr, Phys. Rev. Lett. 117, 087205 (2016).

[23] C. E. Graves et al., Nat. Mater. 12, 293 (2013).

[24] I. Razdolski, A. Alekhin, N. Ilin, J. P. Meyburg, V. Roddatis, D. Diesing, U. Bovensiepen, and A. Melnikov, Nat. Commun. 8, 15007 (2017).

[25] V. E. Demidov, S. Urazhdin, E. R. J. Edwards, M. D. Stiles, R. D. McMichael, and S. O. Demokritov, Phys. Rev. Lett. 107, 107204 (2011).

[26] S. Petit, C. Baraduc, C. Thirion, U. Ebels, Y. Liu, M. Li, P. Wang, and B. Dieny, Phys. Rev. Lett. 98, 077203 (2007).

[27] M. N. Baibich, J. M. Broto, A. Fert, F. N. Van Dau, F. Petroff, P. Etienne, G. Creuzet, A. Friederich, and J. Chazelas, Phys. Rev. Lett. 61, 2472 (1988).

[28] C. Kittel, Phys. Rev. 73, 155 (1948).

[29] G. E. W. Bauer, Y. Tserkovnyak, D. Huertas-Hernando, and A. Brataas, Phys. Rev. B 67, 094421 (2003).

[30] See Supplemental Material at http://link.aps.org/ supplemental/10.1103/PhysRevLett.119.257201 for a detailed description of the proposed spin-scattering model, relation between the magnon population and resistance, and simulation of heating effects, which includes Refs. [31-36].

[31] A. Brataas, Y. V. Nazarov, and G. E. W. Bauer, Phys. Rev. Lett. 84, 2481 (2000).

[32] T. Valet and A. Fert, Phys. Rev. B 48, 7099 (1993).

[33] V. Kambersky and C. E. Patton, Phys. Rev. B 11, 2668 (1975).

[34] F. Menzinger, G. Caglioti, G. Shirane, R. Nathans, S. J. Pickart, and H. A. Alperin, J. Appl. Phys. 39, 455 (1968).

[35] J. Walowski, M. D. Kaufmann, B. Lenk, C. Hamann, J. McCord, and M. Mnzenberg, J. Phys. D 41, 164016 (2008).

[36] M. Djordjevic(to be published).

[37] L. Spietz, K. W. Lehnert, I. Siddiqi, and R. J. Schoelkopf, Science 300, 1929 (2003).

[38] Y. Wang and L. J. Sham, Phys. Rev. B 85, 092403 (2012).

[39] Y. Wang and L. J. Sham, Phys. Rev. B 87, 174433 (2013).

[40] Y. Wang, Y. Zhou, and F.-C. Zhang, Appl. Phys. Lett. 103, 022403 (2013).

[41] S. Urazhdin, Phys. Rev. B 69, 134430 (2004).

[42] M. L. Polianski and P. W. Brouwer, Phys. Rev. Lett. 92, 026602 (2004).

[43] T. Jungwirth, X. Marti, P. Wadley, and J. Wunderlich, Nat. Nanotechnol. 11, 231 (2016).

[44] Y. Tserkovnyak, A. Brataas, and G. E. W. Bauer, Phys. Rev. Lett. 88, 117601 (2002).

[45] K. Ando, S. Takahashi, K. Harii, K. Sasage, J. Ieda, S. Maekawa, and E. Saitoh, Phys. Rev. Lett. 101, 036601 (2008).

[46] L. Liu, C.-F. Pai, Y. Li, H. W. Tseng, D. C. Ralph, and R. A. Buhrman, Science 336, 555 (2012).

[47] H. Nakayama, M. Althammer, Y.-T. Chen, K. Uchida, Y. Kajiwara, D. Kikuchi, T. Ohtani, S. Geprägs, M. Opel, S. Takahashi, R. Gross, G. E. W. Bauer, S. T. B. Goennenwein, and E. Saitoh, Phys. Rev. Lett. 110, 206601 (2013).

[48] E. Turgut, D. Zusin, D. Legut, K. Carva, R. Knut, J. M. Shaw, C. Chen, Z. Tao, H. T. Nembach, T. J. Silva, 
S. Mathias, M. Aeschlimann, P. M. Oppeneer, H. C. Kapteyn, M. M. Murnane, and P. Grychtol, Phys. Rev. B 94, 220408 (2016).

[49] I. Razdolski, A. Alekhin, N. Ilin, J. P. Meyburg, V. Roddatis, D. Diesing, U. Bovensiepen, and A. Melnikov, Nat. Commun. 8, 15007 (2017).

[50] D. Rudolf, C. La-O-Vorakiat, M. Battiato, R. Adam, J. M. Shaw, E. Turgut, P. Maldonado, S. Mathias, P. Grychtol, H. T. Nembach, T. J. Silva, M. Aeschlimann, H. C. Kapteyn, M. M. Murnane, C. M. Schneider, and P. M. Oppeneer, Nat. Commun. 3, 1037 (2012).
[51] M. Hatami, G. E. W. Bauer, Q. Zhang, and P. J. Kelly, Phys. Rev. Lett. 99, 066603 (2007).

[52] J. Foros, A. Brataas, Y. Tserkovnyak, and G. E. W. Bauer, Phys. Rev. Lett. 95, 016601 (2005).

[53] J.-E. Wegrowe, Solid State Commun. 150, 519 (2010).

[54] Z. Zhang, L. Bai, X. Chen, H. Guo, X. L. Fan, D. S. Xue, D. Houssameddine, and C.-M. Hu, Phys. Rev. B 94, 064414 (2016).

[55] G.-M. Choi, C.-H. Moon, B.-C. Min, K.-J. Lee, and D. G. Cahill, Nat. Phys. 11, 576 (2015). 